\title{
Lack of Effect of SU1498, an Inhibitor of Vascular Endothelial Growth Factor Receptor-2, in a Transgenic Murine Model of Retinoblastoma
}

\author{
C.M. Cebulla, M.E. Jockovich, H. Boutrid, Y. Piña, M. Ruggeri, S. Jiao, S.K. Bhattacharya, \\ W.J. Feuer and T.G. Murray*
}

Bascom Palmer Eye Institute, Department of Ophthalmology, University of Miami Miller School of Medicine, Miami,
Florida, USA

\begin{abstract}
SU1498, a tyrosine kinase inhibitor of vascular endothelial growth factor receptor 2 (VEGFR-2), has activity against retinal neovascular diseases. To determine if this drug might have clinical utility against retinoblastoma, we evaluated the effects of SU1498, as well as the expression of VEGFR-2, in a transgenic animal model of retinoblastoma. Optical coherence tomography (OCT) was evaluated as a technology to measure retinal tumors in vivo, in response to treatment. Immunofluorescence analysis was performed to evaluate the distribution and expression of VEGFR-2 in enucleated eyes from LHBTag transgenic mice and controls at 4, 8, 12, and 16 weeks of age. VEGFR-2 and phosphorylated (p)VEGFR-2 levels were quantitated by Western blot. OCT was used to pair 10-week-old animals based on tumor volume $(\mathrm{n}=10)$, and these animals were treated with 6 periocular injections of SU1498 (50mg/kg, given twice weekly) or vehicle for 3 weeks. Tumor burden was determined by histology and in vivo imaging by OCT. VEGFR-2 and pVEGFR-2 expression levels were upregulated during tumorigenesis. However, SU1498 did not significantly reduce tumor burden compared to vehicle $(\mathrm{p}=0.29)$. OCT imaging of one matched pair demonstrated equivalent, linear tumor growth despite treatment with SU1498. Retinal tumors can be followed non-invasively and quantitatively measured with OCT. VEGFR-2 is strongly upregulated during tumorigenesis in transgenic retinoblastoma; however, SU1498 does not decrease tumor volume in transgenic murine RB at the studied dose and route of administration.
\end{abstract}

\section{INTRODUCTION}

Retinoblastoma generates a robust angiogenic response important for its growth and survival [1-5]. Investigating the mechanisms of this response is a major goal for developing new adjuvant therapies for retinoblastoma.

Using the LHßTag transgenic mouse model of retinoblastoma, we have shown that tumor burden is significantly decreased by two independent, anti-angiogenic treatments, combretastatin A-4 [6] and anecortave acetate [7]. Evaluating novel anti-angiogenic agents with different mechanisms of action is a promising strategy, as multiple drugs may eventually be combined for a more robust effect.

VEGFR-2 (also known as KDR or FLK-1) is a high affinity tyrosine kinase receptor for VEGF, known to be very important in mediating normal and pathologic angiogenic responses, especially in cancer [2, 8-14]. Recently, antiangiogenic drugs which inhibit VEGFR have been developed which have shown promise in treating a variety of cancers [15-21]. One promising drug is SU1498, a tyrosine kinase inhibitor specific for VEGFR-2 [22]. Saishin et al. previously showed that SU1498 blocks retinal vascular leakage mediated by VEGF in a murine model [23]. With these novel therapies, it is important to confirm the tumor, or its microenvironment, express the molecules targeted by the therapy. Stitt et al. demonstrated previously that human retinoblastoma expresses VEGFR-2 [2].

\footnotetext{
*Address correspondence to this author at the Bascom Palmer Eye Institute, Department of Ophthalmology, University of Miami Miller School of Medicine, Miami, Florida, USA; E-mail: tmurray@med.miami.edu
}

The LH $\beta$ Tag mouse model of retinoblastoma is a transgenic mouse that expresses the SV40 Large T antigen. This protein sequesters $\mathrm{p} 53$ and $\mathrm{pRB}$ resulting in heritable, bilateral, multifocal retinal tumors that initiate in the inner nuclear layer and are histologically similar to human retinoblastoma [24-29]. Although, the mechanism of tumor initiation between human RB and LHßTag retinal tumors is different, this model is one of the best preclinical models to evaluate novel anti-retinoblastoma therapies. Herein, we tested the hypothesis that VEGFR-2 is upregulated and phosphorylated in transgenic murine $\mathrm{RB}$ and that the VEGFR-2 inhibitor SU1498 decreases tumor burden. OCT imaging was used to evaluate the tumor response to therapy in vivo.

\section{MATERIALS AND METHODOLOGY}

\section{Animals}

The study protocol was approved by the University of Miami, School of Medicine Animal Care and Use Review Board, Miami, FL. All experiments in this study were conducted in accordance with the Association for Research in Vision and Ophthalmology guidelines for the use of animals in ophthalmologic and vision research. Transgenic mice positive for the LH $\beta$ Tag construct have been previously described, and were identified through polymerase chain reaction analysis of tail DNA [24-29].

\section{Immunofluorescence Analysis}

Transgenic and background control mice were sacrificed with $\mathrm{CO}_{2}$ gas at $4,8,10-12$, or 16 weeks ( $\mathrm{n}=4$ per group). Eyes were enucleated, snap frozen in optimal cutting temperature compound (Tissue Tek), and sectioned serially (5 
microns). For immunofluorescence, slides were fixed with cold methanol 15 minutes at $-20^{\circ} \mathrm{C}$. Blocking solution (phosphate buffered saline (PBS) with $5 \%$ bovine serum albumin, and $1 \%$ Triton-X 100) was added to the slides for 1 hour at room temperature and drained off. VEGFR-2 was detected with a rat monoclonal antibody (clone 4H3B6H9, Chemicon International, Temecula, CA) and Alexa Fluor 488conjugated secondary antibody (Invitrogen). As a control, the primary antibody was omitted. DAPI (Invitrogen) was used as a counterstain. Slides were coverslipped with Antifade mounting media (Biomeda, Foster City, CA).

Twelve to 16 sections per eye were viewed with an Olympus Bx51 fluorescent microscope and images were digitally acquired. Exposure times for VEGFR-2 immunofluorescence were standardized within each experiment.

\section{Histologic Tumor Burden Analysis}

Tumor burden was determined by standard hematoxylin and eosin-(H\&E) as previously described [30]. Four of every 6 frozen sections of the eye were fixed in $4 \%$ paraformaldehyde and stained with H\&E. All stained sections were evaluated for the largest tumor area at a magnification of $40 \mathrm{X}$. Digital photographs were acquired and the tumor area was calculated and normalized to the area of the globe [30].

\section{Western Blot}

Retinas and retinal tumors ( $\mathrm{n}=8$ per group) from 4,8 , and 16 week-old LH $\beta$ Tag mice and background controls were removed under a dissecting microscope using a \#10 blade scalpel and iris spatula (FST Instruments). Non-retinal structures were removed with jewelers' forceps. Retinas and tumors were pooled and homogenized with cold extraction buffer (125 mM TrisCl pH 7.0, $100 \mathrm{mM} \mathrm{NaCl}$ and $1 \%$ Sodium dodecyl sulphate (SDS)). Proteins were quantitated using the Bradford method. Equal microgram quantities of protein were suspended in Laemmli buffer and fractionated with SDS-PAGE on 4-20\% precast gels (Invitrogen). Recombinant human VEGFR-2-Fc chimera (R\&D Systems) was used as a positive control for VEGFR-2 and pVEGFR-2 proteins. Proteins were electroblotted onto a polyvinylidene fluoride (PVDF) membrane. Membranes were blocked with $5 \%$ nonfat milk and incubated with VEGFR-2 primary antibody (FLK-1 (C-1158), Santa Cruz) or pVEGFR2 primary antibody (PhosphoDetect Anti-VEGFR2 (pTyr1214), Calbiochem) and a secondary antibody conjugated to horseradish peroxidase. Blots were developed using ECL chemiluminescence. Blots were stripped and re-probed for $\beta$-actin (clone C4, Santa Cruz) as a loading control.

\section{OCT Analysis}

A spectral-domain optical coherence tomography (OCT) system for noninvasive, in vivo, high resolution imaging of rodent retina was used as previously described [31]. The configuration of the OCT system used in this study is similar to the previous study except that the depth resolution was improved to $\sim 3 \mu \mathrm{m}$ in tissue. The right eyes of $10 \mathrm{LH} \beta \mathrm{Tag}$ mice were imaged in vivo, and the mice were paired according to greatest cross-sectional tumor width: small $(<1 / 3 \mathrm{~mm})$, medium $(1 / 3 \mathrm{~mm}$ to $<3 / 4 \mathrm{~mm})$, medium-large $(3 / 4 \mathrm{~mm}$ to $<1 \mathrm{~mm})$, large $(1 \mathrm{~mm}$ to $<1.5 \mathrm{~mm})$, and very large $(\geq 1.5 \mathrm{~mm})$.
For one select pair (animal numbers II8 and MM8), the tumor location was registered and volume was extracted once a week for 3 weeks (time 0 , week 1 , week 2 , and week 3 postinjection \#1). A semiautomatic segmentation tool for segmenting the tumor boundaries in each OCT cross-sectional image was used. The tumor volume was then calculated automatically using the segmented boundaries.

\section{SU1498 Experiments}

Spectral OCT was used to pair 10-week-old LHßTag mice based on tumor volume $(\mathrm{n}=10)$. Paired animals were treated with 6 periocular injections (given in a $10 \mu 1$ volume twice weekly for 3 weeks) of either SU1498 $(50 \mathrm{mg} / \mathrm{kg}$, LC Labs, $n=5)$ or DMSO vehicle $(n=5)$. Mice were sacrificed 4 days after the final injection. Tumor burden was determined by H\&E histology and weekly, in vivo imaging by OCT. The dose of SU1498 was based on studies by Saishin et al. [23], which demonstrated prevention of retinal leakage from intravitreal injection of VEGF in C57BL/6J mice. A similar study without prior OCT selection was also performed by this laboratory in which SU1498 $(50 \mathrm{mg} / \mathrm{kg})$ was given via oral gavage. In this study, 6 doses of SU1498 (or vehicle) were delivered to 10 week LH $\beta$ Tag mice ( $\mathrm{n}=5$ per group) twice weekly for 3 weeks. Mice were enucleated at 16 weeks of age, and eyes were formalin-fixed, paraffin-embedded, sectioned, and tumor burden was analyzed by H\&E staining.

\section{RESULTS}

To test the hypothesis that VEGFR-2 expression is upregulated during tumorigenesis in the LH $\beta$ Tag transgenic model of retinoblastoma, we performed immunofluorescence analyses. LHßTag mice develop multiple, bilateral retinal tumors that are typically not visible on histology at 4 weeks of age, small at 8 weeks, medium-sized at 10-12 weeks, and fill the globe by 16 weeks of age [30]. Eyes from 4, 8, 10-12, and 16 week-old transgenic mice and background controls were evaluated ( $\mathrm{n}=4$ per group). In eyes with medium and large tumors, VEGFR-2 expression could be seen in 4 week positive mice in the ganglion cell layer and flanking the inner nuclear layer, but overall levels were very low. In contrast, VEGFR-2 levels were strongly upregulated in 8, 10-12, and 16 week transgenic mice, both in areas of tumor and in the retina (Fig. 1). VEGFR-2 immunostaining was most intense in the ganglion cell layer, inner plexiform layer, outer plexiform layer, and within the body of tumors. Large tumors (16 week) had VEGFR-2 immunofluorescence throughout the tumor (Fig. 1). Immunohistochemical analyses suggest that VEGFR-2 is expressed by Muller glia as well as endothelial cells in this tumor. VEGFR-2 staining colocalizes with molecules associated with Muller glia (CRALBP and vimentin) more than with endothelial cells (CD105 and lectin, data not shown).

In order to confirm the upregulation of VEGFR-2 during tumorigenesis, we performed Western blot analyses on retinas and tumors isolated from 4,8 , and 16 week LH $\beta$ Tag mice and background controls ( $\mathrm{n}=8$ retinas per group). VEGFR-2 levels were elevated in 8 and 16 week old transgenic mice compared with 4 week old mice and negative controls (Fig. 2). The activation of VEGFR-2 begins with VEGF binding the receptor, leading to its phosphorylation [10]. Western blot analysis of pVEGFR-2 levels was 

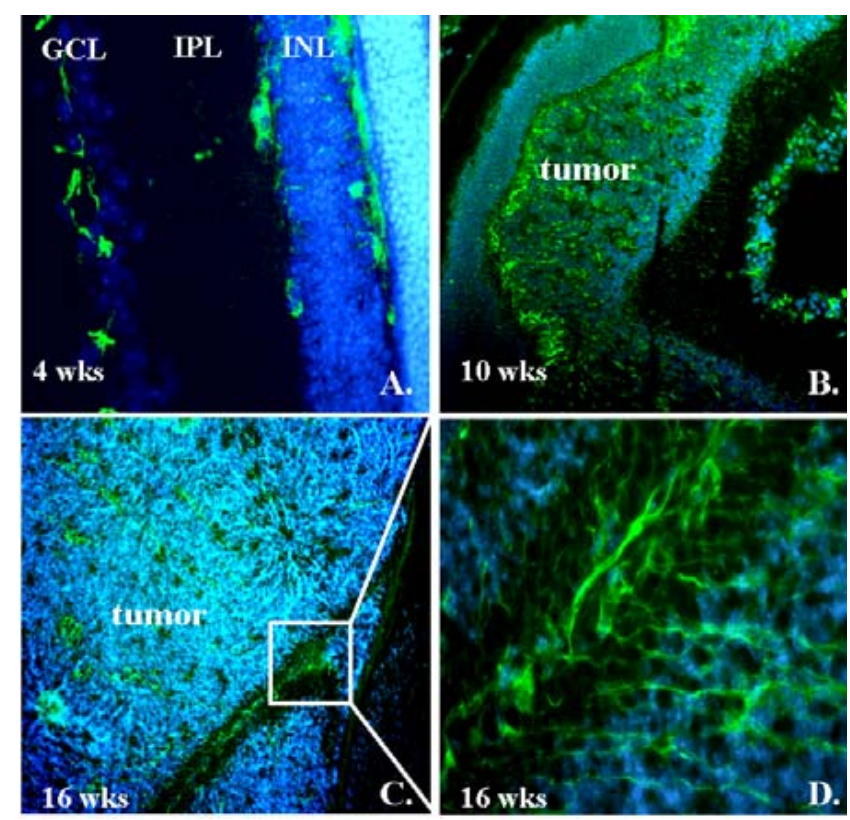

Fig. (1). VEGFR-2 immunofluorescence staining in LH $\beta$ Tag transgenic retinoblastoma. Representative eyes from LHßTag mice at 4 (A), 10 (B), and 16 (C,D) weeks are shown. VEGFR-2 immunoreactivity is green and DAPI counterstaining is blue. GCL, ganglion cell layer; IPL, inner plexiform layer; INL, inner nuclear; ONL, Outer nuclear layer. Magnification (A) $200 \mathrm{X}$, (B) $100 \mathrm{X}$, (C) $200 \mathrm{X}$. (D) zoomed image from inset of C.

performed to confirm that VEGFR-2 phosphorylation occurs in transgenic retinoblastoma. Levels of pVEGFR-2 were elevated in 4- and 16-week-old LHßTag retinal and tumor extracts compared to controls (Fig. 2). Immunoblots were probed for $\beta$-actin as a loading control (Fig. 2).

Since VEGFR-2 is upregulated and phosphorylated in transgenic retinoblastoma, we hypothesized that pharmacologically blocking VEGFR-2 would be an effective therapeutic strategy, reducing tumor burden. To test this hypothesis, LH $\beta$ Tag mice ( $\mathrm{n}=5$ per group) were treated with SU1498, a drug shown to effectively block retinal leakage in response to VEGF intravitreal injections in mice [23]. The same dose $(50 \mathrm{mg} / \mathrm{kg})$ was used in this study, and the drug was delivered to 10 week transgenic mice via $6,10 \mathrm{ul}$ periocular injections twice weekly for 3 weeks. The vehicle, DMSO, was used as a control. Mice were first paired based on tumor size, as determined with spectral OCT imaging. The tumors were grouped into small, medium, medium-large, and large sizes as described in the methods (Table 1). Side-effects of both DMSO and SU1498 included moderately severe orbital fibrosis in all animals. Other side effects included corneal abrasions and ulceration, conjunctival hyperemia, and neovascularization of the cornea in one animal. Abrasions and ulcerations were treated with erythromycin ophthalmic ointment. No infection or corneal perforations occurred.

As shown in Fig. (3A), the tumor burden was decreased after treatment with SU1498 compared with DMSO control, but not significantly ( $p=0.29$, paired $t$-test). A $95 \%$ confidence interval around the non-significant mean difference of DMSO - SU1498 tumor to globe ratio (0.06) ranged from 0.08 to +0.19 . Similarly, analysis of each pair shows no significant improvement in SU1498 treated eyes compared to
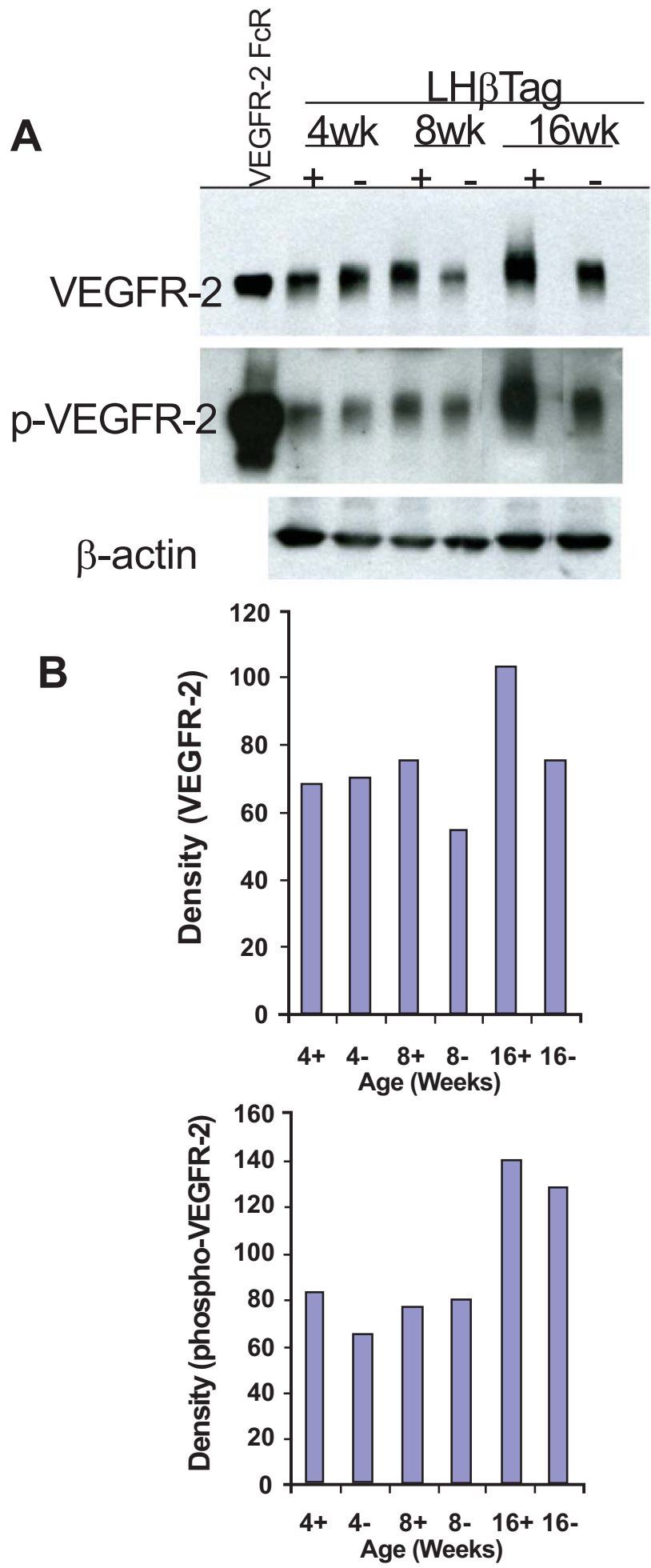

Fig. (2). VEGFR-2 and pVEGFR-2 are upregulated in transgenic RB. (A) Western blot analysis was performed in retinal/tumor isolates from LH $\beta$ Tag mice and controls at 4,8 , and 16 weeks of age $(\mathrm{n}=8$ pooled retinas per group). Actin was probed as a loading control. One representative blot is shown of 3 replicates. (B) Graphic representation of one representative experiment showing quantitative changes in VEGFR-2 and pVEGFR-2 levels (in relative units) from Western blot analysis. Animal age (weeks) and tumor status ( + for positive or - for negative tumor burden) is indicated on the $\mathrm{x}$ axis. 
Table 1. LH $\beta$ Tag Mice were Paired into Groups with Equivalent Tumor Burden Prior to Initiating Therapy. The Final Tumor Areas (in Pixels, Determined from Histology) After Treatment are Shown

\begin{tabular}{|l|c|c|c|}
\hline \multicolumn{1}{|c|}{ Pair Number } & Initial Tumor Size & Final Tumor Area (SU1498) & Final Tumor Area (DMSO) \\
\hline \hline 1 (II5:LL3) & Very Large & 137,509 & 169,258 \\
\hline 2 (II6: JJ9) & Large & 69,321 & 22,721 \\
\hline 3 (LL1-LL2) & Large & 54,585 & 324,610 \\
\hline 4 (II8:MM8) & Medium & 74,099 & 78,967 \\
\hline 5 (KK1:KK3) & Medium & 85,129 & 67,897 \\
\hline Average & & $\mathbf{8 4 , 1 2 9}$ & $\mathbf{1 3 2 , 6 9 1}$ \\
\hline Standard dev. & & $\mathbf{3 1 , 7 9 3}$ & $\mathbf{1 1 9 , 7 3 7}$ \\
\hline
\end{tabular}
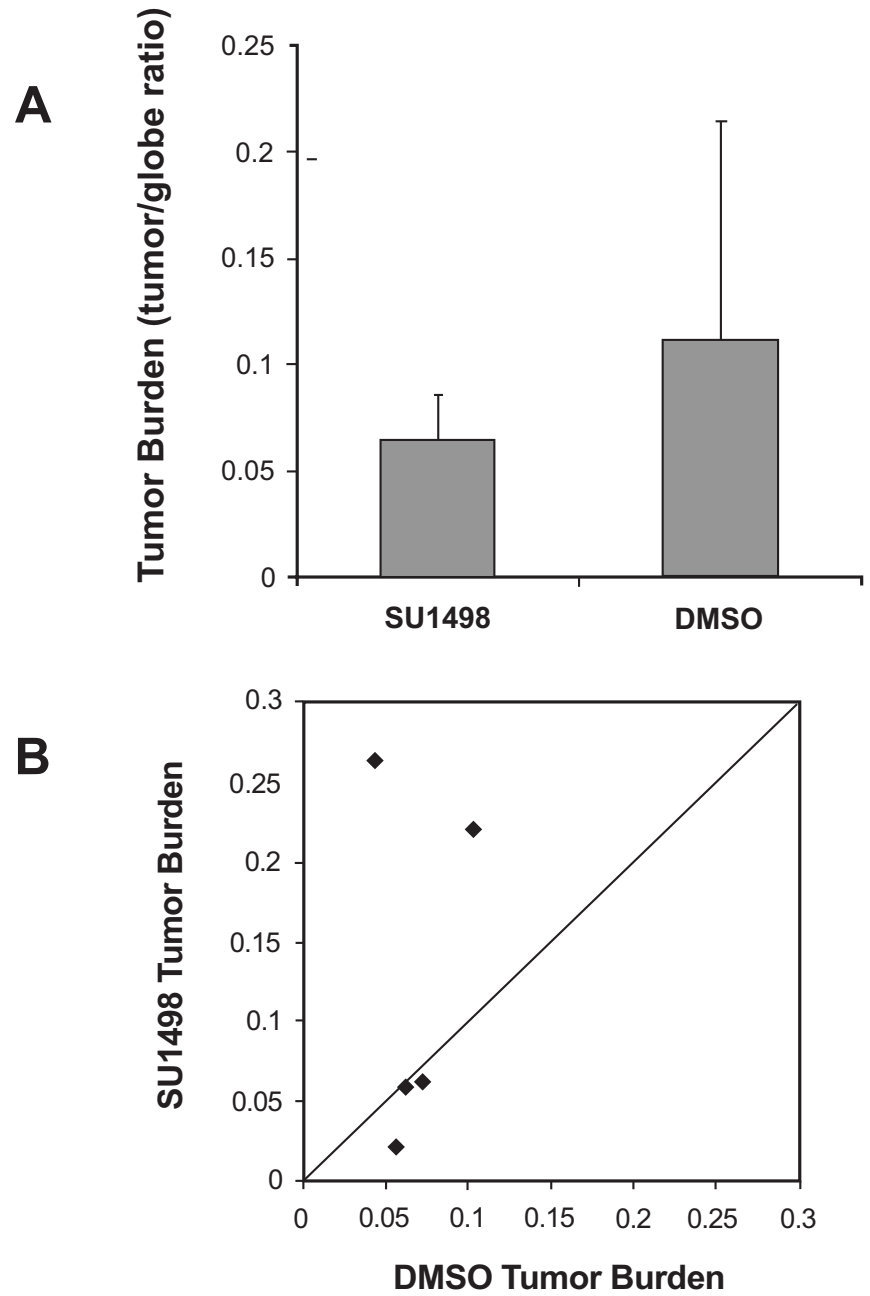

Fig. (3). VEGFR-2 inhibitor SU1498 did not significantly reduce tumor burden. (A) Histologic analysis demonstrates that the average tumor burden (tumor/globe ratio) of LH $\beta$ Tag right eyes was not significantly decreased by six periocular injections of SU1498, compared with DMSO control. (B) Scatter plot of tumor burden in DMSO-treated versus SU1498-treated eyes. Each point represents the tumor burden of one pair, with DMSO tumor burden on the Yaxis and SU1498 tumor burden on the X-axis. The diagonal line indicates equal tumor burden in the SU1498-treated eyes and DMSO control. Pairs in which SU1498-treated eyes had smaller tumors appear in the upper triangle of the graph. controls, although in two pairs the tumor burden was substantially less in SU1498-treated eyes (Fig. 3B). These negative data were corroborated by another study in which SU1498 $(50 \mathrm{mg} / \mathrm{kg})$ was delivered to LH $\beta$ Tag mice via oral gavage instead of periocular injection $(n=5$ SU1498 and 5 vehicle controls (data not shown)).

Spectral OCT technology has now enabled non-contact, in vivo imaging of LH $\beta$ Tag retinal tumor response to drug therapies. Two paired mice (study number II8 and MM8) were imaged once each week during the course of the experiment, and their tumors were followed. Both SU1498 and DMSO treated animals showed linear increases in tumor volume during the 2 weeks evaluated, with no significant differences (Fig. 4). By the third week of the experiment (age 13 weeks), the tumor volume was not measured since tumor size exceeded the detection boundaries of this system (data not shown). The calculated tumor volumes (in cubic millimeters) are shown in Table 2.

\section{DISCUSSION}

Herein we show that although VEGFR-2 is upregulated and phosphorylated in transgenic murine retinoblastoma during tumorigenesis, treatment with the VEGFR-2 blocking drug SU1498 does not significantly decrease tumor burden at the dose studied, even though SU1498 tumor burden was substantially less in two animal pairs. To our knowledge, this is the first study to (1) pair animals with equivalent ocular tumor burden, in a transgenic model, and (2) follow tumor burden response to drug therapy in vivo, with OCT technology.

The dose of SU1498 was carefully selected (a dose with known in vivo activity in mice [23]) and was delivered by both periocular injection and oral gavage. In contrast, two other anti-angiogenic drugs: anecortave acetate and combretastatin A-4, both significantly impacted transgenic retinoblastoma tumor volume [6,7].

Of VEGF receptors, VEGFR-2 is most important in mediating angiogenesis; alone it is sufficient to mediate all of the angiogenic responses to VEGF [19,32]. Tumor growth has been successfully inhibited by manipulating VEGFR-2 activity, by using a dominant negative mutant of VEGFR-2 [15] and antibodies that block VEGF activity [20,21]. Further, novel anti-tyrosine kinase drugs specific for VEGF receptors, including PTK787/ZK 222584, ZD 6474, and 
Table 2. Tumor Volume Calculations, in Cubic Millimeters, from Spectral OCT Imaging

\begin{tabular}{|c|c|c|c|}
\hline Pair Number & Tumor Volume Week 0 & Tumor Volume Week 1 & Tumor Volume Week 2 \\
\hline \hline II8 (SU1498) & 0.0338 & 0.0540 & 0.0697 \\
\hline MM8 (DMSO) & 0.0267 & 0.0507 & 0.0712 \\
\hline
\end{tabular}

A

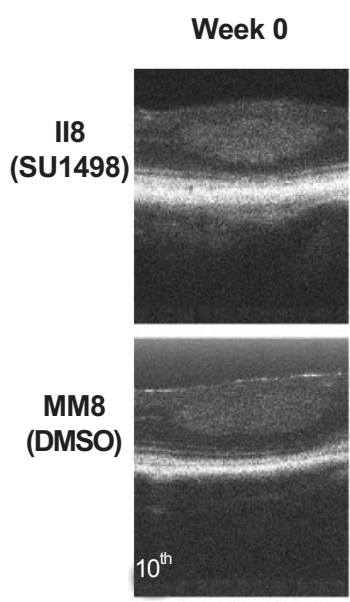
Time Post-Injection Week $1 \quad$ Week 2
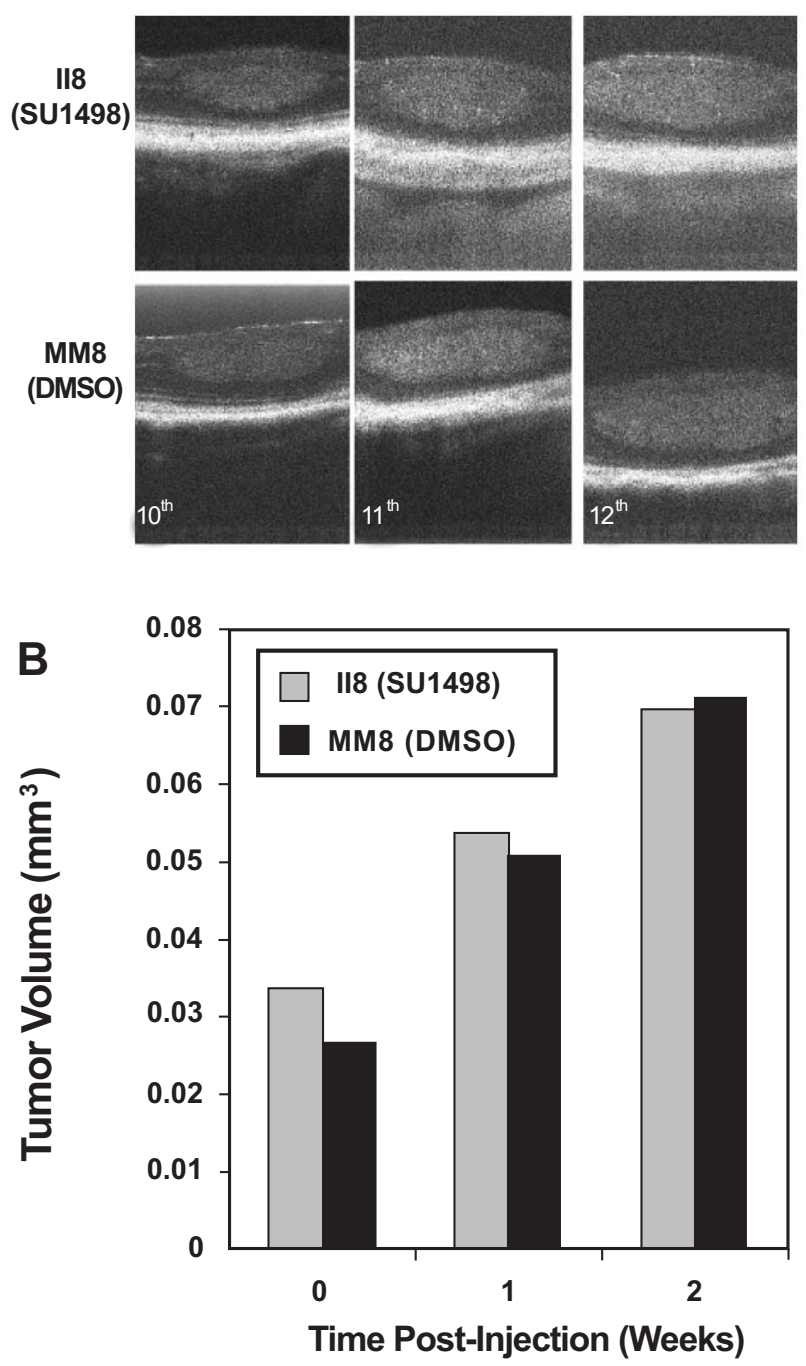

Fig. (4). OCT effectively imaged tumor burden changes in response to SU1498 treatment. In vivo imaging was performed on a registered tumor in one matched pair of LH $\beta$ Tag mice. (A) Pictures of the tumor in cross-section are shown prior to treatment (time 0 , age 10 weeks), 1 week post-injection number 1 (11 weeks), and 2 weeks post injection number 1 (12 weeks). (B) Graph of the tumor volume determined by OCT segmentation methods.

SU5416, have shown promise in animal models and phase I/II trials for several cancers [16-18,21,33]. However, tumors may be able to make several different angiogenic factors as they develop; allowing compensation for the inactivation of one pro-angiogenic pathway (e.g., tumor bFGF levels increased in breast tumors when VEGF expression was elimi- nated) [34]. Thus, adding an anti-angiogenic drug with a different mechanism (such as endostatin) to VEGFR-2 inhibitors may significantly improve efficacy, as shown by Abdollahi et al. [35].

Anecortave acetate, an angiostatic cortisene, blocks angiogenesis by inhibiting matrix metalloproteinases and urokinase plasminogen activator [36]. Combretastatin A-4 is a microtubule-binding agent which targets angiogenic vessels, in part by disrupting vascular endothelial-cadherin signaling [37]. It is tempting to speculate that the mechanisms of these drugs are more far-reaching in inhibiting the multifaceted angiogenic responses of transgenic retinoblastoma tumors. In fact, inhibiting multiple classes of VEGF receptors or all VEGF isoforms may have a greater efficacy against transgenic retinoblastoma tumors. Recently, Gille et al. [38] showed no significant impact on metastatic melanoma tumor burden when just VEGFR-1 or -2 was targeted, but a significant reduction in tumor burden was achieved when both treatments were combined.

In summary, targeting the angiogenic response of retinoblastoma tumors seems to be a promising therapeutic strategy. However, significant testing of combination strategies may be necessary to find the most efficacious therapies.

\section{ACKNOWLEDGEMENTS}

We would like to thank Gabe Gaidosh for confocal microscopy assistance and Magda Celdran for excellent histology. We thank Dr. George Hoppe for helpful comments on Western blot. This work was supported by NIH RO1 EY013629, NIH P30-EY014801, and Research to Prevent Blindness.

\section{REFERENCES}

[1] Tapper D, Langer R, Bellows AR, Folkman J. Angiogenesis capacity as a diagnostic marker for human eye tumors. Surgery 1979; 86: 36-40.

[2] Stitt AW, Simpson DA, Boocock C, Gardiner TA, Murphy GM, Archer DB. Expression of vascular endothelial growth factor (VEGF) and its receptors is regulated in eyes with intra-ocular tumours. J Pathol 1998; 186: 306-312.

[3] Marback EF, Arias VE, Paranhos A, Jr., Soares FA, Murphree AL, Erwenne CM. Tumour angiogenesis as a prognostic factor for disease dissemination in retinoblastoma. Br J Ophthalmol 2003; 87: 1224-1228.

[4] Rossler J, Dietrich T, Pavlakovic H, et al. Higher vessel densities in retinoblastoma with local invasive growth and metastasis. Am J Pathol 2004; 164: 391-394.

[5] Pe'er J, Neufeld M, Baras M, Gnessin H, Itin A, Keshet E. Rubeosis iridis in retinoblastoma. Histologic findings and the possible role of vascular endothelial growth factor in its induction. Ophthalmology 1997; 104: 1251-1258.

[6] Escalona-Benz E, Jockovich M-E, Murray TG, et al. Combretastatin a-4 prodrug in the treatment of a murine model of retinoblastoma. Invest Ophthalmol Vis Sci 2005; 46: 8-11.

[7] Jockovich ME, Murray TG, Escalona-Benz E, Hernandez E, Feuer W. Anecortave acetate as single and adjuvant therapy in the treatment of retinal tumors of $\mathrm{LH}($ beta)T(ag) mice. Invest Ophthalmol Vis Sci 2006; 47: 1264-1268. 
[8] Veikkola T, Karkkainen M, Claesson-Welsh L, Alitalo K. Regulation of angiogenesis via vascular endothelial growth factor receptors. Cancer Res 2000; 60: 203-212.

[9] Matsumoto T, Claesson-Welsh L. VEGF receptor signal transduction. Sci STKE 2001; 2001: re21-

[10] Ferrara N, Gerber H-P, LeCouter J. The biology of VEGF and its receptors. Nat Med 2003; 9: 669-676.

[11] Stone J, Itin A, Alon T, et al. Development of retinal vasculature is mediated by hypoxia-induced vascular endothelial growth factor (VEGF) expression by neuroglia. J Neurosci 1995; 15: 4738-4747.

[12] Sakurai Y, Ohgimoto K, Kataoka Y, Yoshida N, Shibuya M. Essential role of flk-1 (VEGF receptor 2) tyrosine residue 1173 in vasculogenesis in mice. Proc Natl Acad Sci USA 2005; 102: 10761081.

[13] Witmer AN, Blaauwgeers HG, Weich HA, Alitalo K, Vrensen GFJM, Schlingemann RO. Altered expression patterns of VEGF receptors in human diabetic retina and in experimental VEGFinduced retinopathy in monkey. Invest Ophthalmol Vis Sci 2002; 43: 849-857.

[14] Schweigerer L. Antiangiogenesis as a novel therapeutic concept in pediatric oncology. J Mol Med 1995; 73: 497-508.

[15] Millauer B, Longhi MP, Plate KH, et al. Dominant-negative inhibition of flk-1 suppresses the growth of many tumor types in vivo. Cancer Res 1996; 56: 1615-1620.

[16] Hess-Stumpp H, Haberey M, Doz P, Thierauch K-H. PTK 787/zk 222584, a tyrosine kinase inhibitor of all known VEGF receptors, represses tumor growth with high efficacy. ChemBioChem 2005; 6: 550-557.

[17] Goldbrunner RH, Bendszus M, Wood J, Kiderlen M, Sasaki M, Tonn JC. Ptk787/zk222584, an inhibitor of vascular endothelial growth factor receptor tyrosine kinases, decreases glioma growth and vascularization. Neurosurgery 2004; 55: 426-432; discussion 432.

[18] Peterson AC, Swiger S, Stadler WM, Medved M, Karczmar G, Gajewski TF. Phase II study of the flk-1 tyrosine kinase inhibitor SU5416 in advanced melanoma. Clin Cancer Res 2004; 10: 40484054.

[19] Witte L, Hicklin DJ, Zhu Z, et al. Monoclonal antibodies targeting the VEGF receptor-2 (flk1/kdr) as an anti-angiogenic therapeutic strategy. Cancer Metastasis Rev 1998; 17: 155-161.

[20] Prewett M, Huber J, Li Y, et al. Antivascular endothelial growth factor receptor (fetal liver kinase 1) monoclonal antibody inhibits tumor angiogenesis and growth of several mouse and human tumors. Cancer Res 1999; 59: 5209-5218.

[21] Brazelle WD, Shi W, Siemann DW. VEGF-associated tyrosine kinase inhibition increases the tumor response to single and fractionated dose radiotherapy. Int J Radiat Oncol Biol Phys 2006; 65: 836-841.

[22] Strawn LM, McMahon G, App H, et al. Flk-1 as a target for tumor growth inhibition. Cancer Res 1996; 56: 3540-3545.

[23] Saishin Y, Saishin Y, Takahashi K, et al. Inhibition of protein kinase $\mathrm{c}$ decreases prostaglandin-induced breakdown of the bloodretinal barrier. J Cell Physiol 2003; 195: 210-219.

[24] Windle JJ, Albert DM, O'Brien JM, et al. Retinoblastoma in transgenic mice. Nature 1990; 343: 665-669.
[25] O'Brien JM, Marcus DM, Bernards R, et al. A transgenic mouse model for trilateral retinoblastoma. Arch Ophthalmol 1990; 108: $1145-1151$.

[26] O'Brien JM, Marcus DM, Niffenegger AS, et al. Trilateral retinoblastoma in transgenic mice. Trans Am Ophthalmol Soc 1989; 87: 301-322; discussion 322-306.

[27] Marcus DM, Lasudry JG, Carpenter JL, et al. Trilateral tumors in four different lines of transgenic mice expressing SV40 T-antigen. Invest Ophthalmol Vis Sci 1996; 37: 392-396.

[28] Albert DM, Griep AE, Lambert PF, Howes KA, Windle JJ, Lasudry JG. Transgenic models of retinoblastoma: What they tell us about its cause and treatment. Trans Am Ophthalmol Soc 1994; 92: 385-400; discussion 400-381.

[29] Mills MD, Windle JJ, Albert DM. Retinoblastoma in transgenic mice: Models of hereditary retinoblastoma. Surv Ophthalmol 1999; 43: 508-518.

[30] Jockovich M-E, Bajenaru ML, Pina Y, et al. Retinoblastoma tumor vessel maturation impacts efficacy of vessel targeting in the LHbetaTag mouse model. Invest Ophthalmol Vis Sci 2007; 48: 24762482.

[31] Ruggeri $\mathrm{M}$, Wehbe $\mathrm{H}$, Jiao $\mathrm{S}$, et al. In vivo three-dimensional highresolution imaging of rodent retina with spectral-domain optica coherence tomography. Invest Ophthalmol Vis Sci 2007; 48: 18081814.

[32] Gille H, Kowalski J, Li B, et al. Analysis of biological effects and signaling properties of FLT-1 (VEGFR-1) and KDR (VEGFR-2). A reassessment using novel receptor-specific vascular endothelial growth factor mutants. J Biol Chem 2001; 276: 3222-3230.

[33] Lee L, Sharma S, Morgan B, et al. Biomarkers for assessment of pharmacologic activity for a vascular endothelial growth factor (VEGF) receptor inhibitor, PTK787/ZK 222584 (PTK/ZK): Translation of biological activity in a mouse melanoma metastasis model to phase I studies in patients with advanced colorectal cancer with liver metastases. Cancer Chemother Pharmacol 2006; 57: 761-771.

[34] Yoshiji H, Harris SR, Thorgeirsson UP. Vascular endothelial growth factor is essential for initial but not continued in vivo growth of human breast carcinoma cells. Cancer Res 1997; 57: 3924-3928.

[35] Abdollahi A, Lipson KE, Sckell AZ, et al. Combined therapy with direct and indirect angiogenesis inhibition results in enhanced antiangiogenic and antitumor effects. Cancer Res 2003; 63: 88908898.

[36] Penn JS, Rajaratnam VS, Collier RJ, Clark AF. The effect of an angiostatic steroid on neovascularization in a rat model of retinopathy of prematurity. Invest Ophthalmol Vis Sci 2001; 42: 283-290.

[37] Vincent L, Kermani P, Young LM, et al. Combretastatin A4 phosphate induces rapid regression of tumor neovessels and growth through interference with vascular endothelial-cadherin signaling. J Clin Invest 2005; 115: 2992-3006.

[38] Gille J, Heidenreich R, Pinter A, et al. Simultaneous blockade of vegfr- 1 and vegfr-2 activation is necessary to efficiently inhibit experimental melanoma growth and metastasis formation. Int J Cancer 2007; 120: 1899-1908. 